\title{
Mean Motions In Conditionally Periodic Separable Systems
}

\author{
John P. Vinti
}

(November 10, 1960)

\begin{abstract}
A search of the literature failed to disclose any general statement or proof of a theorem informally current among dynamical astronomers. The present paper gives a proof of the theorem, which states that, in any conditionally periodic separable system the mean frequency $n_{k}$ of any separation coordinate $q_{k}$ is equal to $\nu_{k} \equiv \partial \alpha_{1} / \partial J_{k}$. Here $\alpha_{1}$ is the energy and $J_{k}$ is the $k$ 'th action variable. The proof is carried out for nonsingular Staeckel systems, so that it is applicable to any nonpolar orbit of an artificial satellite, when the potential leads to separability.
\end{abstract}

\section{Introduction}

Conditionally periodic separable systems are commonly illustrated in works on advanced dynamics by the motion of a particle under the joint action of harmonic oscillator forces at right angles. This happens to be a very special case, for which each rectangular coordinate $q_{k}$ has a constant frequency, equal to the corresponding "fundamental frequency", $\nu_{k} \equiv \partial \alpha_{1} / \partial J_{k}$, where $\alpha_{1}$ is the energy and $J_{k}$ the corresponding action variable.

In a more general system of this type, each generalized coordinate $q_{k}$ may have a variable frequency, but it appears to be generally believed among dynamical astronomers that the mean frequency of $q_{k}$ must be equal to $\nu_{k}$, if the conditionally periodic system is separable. Needing to refer to such a theorem in solving a specific problem, I have searched the literature but have found no explicit statement or proof of it. The present paper is an attempt to furnish such a reference, with a proof sufficiently general to be applicable to all the separable problems that may arise in the gravitational theory of the orbit of a satellite of an oblate planet.

Little of the analysis in this paper can claim to be really new. Much of the pertinent material in the literature, however, is discursive, relatively unavailable, and expressed in notations now unfamiliar to most mathematical physicists. Some of it is inadequate, if not incorrect, especially in the treatment of the periodicity of the $q$ 's as functions of the angle variables. Moreover, none of it seems to have been carried out in the shortest and most appropriate way to prove the theorem in question. The present paper attempts to give a concise and correct treatment that will serve this purpose.

It is easy to see why such a theorem should have escaped formal statement and proof. Physicists have not been concerned with mean frequencies of this kind. Dynamical astronomers have been, but ordinarily for nonseparable systems. Until 1957 their only separable problems were the Kepler prob-

\footnotetext{
1 This work was supported by the U.S. Air Force, through the Office of Scientific Research of the Air Research and Development Command.
}

lem $[1]^{2}$ for which the coordinate frequencies are all equal to the constant value $\nu_{1}=\nu_{2}=\nu_{3}$, and the problem of two centers, which has remained a curiosity to be found in Charlier's famous book [2], but without application. Since 1957 various potentials have been suggested, by Sterne [3], by Garfinkel [4], and by the author [5], for the gravitational field of an oblate planet, all of which lead to separability and to intermediary satellite orbits with $\nu_{1} \neq \nu_{2} \neq \nu_{3}$. Since the solution for these orbits is greatly facilitated by knowledge of the mean coordinate frequencies, it now becomes desirable to have a formal and general proof of the theorem.

It is here convenient to discuss briefly the general plan of the paper, without definitions. The difficulty in proving the theorem arises only when the fundamental frequencies $\nu_{1}, \ldots, \nu_{n}$ are incommensurable. If $w_{1}, \ldots, w_{n}$ are the angle variables, the plan is first to show that for a conditionally periodic system there exist infinitely many values of the time, with no upper bound, at which the orbit in $w$-space passes arbitrarily closely to points separated from the initial point $w_{1}(0), \ldots, w_{n}(0)$ by integer intervals $\Delta w_{k}=m_{k}, \quad k=1, \ldots, n$. This fact follows directly from a theorem of Dirichlet, which is easy to understand and to apply.

To convert this result to $q$-space, it is necessary to know that $q_{k}$ is a single-valued, continuous, periodic function of the $w$ 's. To show this the author restricts considerations to nonsingular Staeckel systems, proving that for them each $q_{k}$ is a single-valued differentiable function of $v_{k} \equiv \int d q_{k} / p_{k}$ and each $v_{k}$ of the angle variables $w_{1}, \ldots, w_{n}$. With a careful use of the single-valuedness, the periodic property then follows.

Application of these properties of the $q$ 's as functions of the $w$ 's shows that, at the values of the time mentioned above, the orbit in $q$-space then passes arbitrarily closely to points where each $q_{k}$ would have gone through exactly $m_{k}$ cycles. The proof of the theorem then follows.

\footnotetext{
${ }^{2}$ Figures in brackets indicate the literature references at the end of this paper.
} 


\section{Staeckel Systems}

If $q_{1}, \ldots, q_{n}$ and $p_{1}, \ldots, p_{n}$ denote the generalized coordinates and momenta of a dynamical system of $n$ degrees of freedom, the system is said to be of the Staeckel type $[2,6,7,8,9]$ if the Hamiltonian $H$ is given by

$$
\begin{aligned}
& H=\frac{1}{2} \sum_{k=1}^{n} A_{k}\left(q_{1}, \ldots, q_{n}\right) p_{k}^{2}+V\left(q_{1}, \ldots, q_{n}\right), \\
& A_{k}>0,(k=1, \ldots, n)
\end{aligned}
$$

and if there exist functions $\phi_{i j}\left(q_{i}\right), \psi_{i}\left(q_{i}\right), \quad i, j=1, \ldots$, $n$, such that

$$
\begin{gathered}
A_{k}=M_{k 1} / \operatorname{det}\left(\phi_{i j}\right), \\
V=\sum_{k=1}^{n} \psi_{k}\left(q_{k}\right) A_{k},
\end{gathered}
$$

$M_{k 1}$ being the cofactor of $\phi_{k 1}\left(q_{k}\right)$ in the determinant $\operatorname{det}\left(\phi_{i j}\right)$.

Conditions (2) and (3) are necessary and sufficient for the separability of a system with such a Hamiltonian.

If we next define the domain $Q$ of the $q$ 's as the totality of real values of $q_{1}, \ldots, q_{n}$ for which $p_{k}^{2} \geqq 0, k=1, \ldots ., n$, we may then define a nonsingular Staeckel system as one for which $\psi_{k}\left(q_{k}\right)$ and $\phi_{k i}\left(q_{k}\right), i, k=1, \ldots, n$, exist and are single-valued and for which det $\left(\phi_{i j}\right) \neq 0$ anywhere in $Q$. If we put

$$
\Phi \equiv\left(\phi_{i j}\left(q_{i}\right)\right)
$$

for the Staeckel matrix, then $\Phi^{-1}$ exists and is singlevalued anywhere in $Q$; in particular,

$$
\left(\Phi^{-1}\right)_{1 k}=A_{k} \quad(k=1, \ldots, n)
$$

all exist anywhere in $Q$. (This restriction thus rules out polar orbits from consideration if the right ascension $\varphi$ is one of the coordinates, since $A_{3}$ then becomes infinite on the polar axis.)

The momenta $p_{k}$ are then given by

$$
p_{k}^{2}=-2 \psi_{k}\left(q_{k}\right)+2 \sum_{i=1}^{n} \phi_{k i}\left(q_{k}\right) \alpha_{i}, \quad(k=1, \ldots, n),
$$

where the $\alpha$ 's are separation constants, $\alpha_{1}$ being the energy. (For satellite problems, where $n=3,2 \alpha_{2}$ and $2 \alpha_{3}$ are usually denoted by $\alpha_{2}^{2}$ and $\alpha_{3}^{2}$.)

The Hamilton-Jacobi function $W$ is then given by

$$
\begin{aligned}
W & =\sum_{k=1}^{n} \int_{q_{k 0}}^{q_{k}} p_{k} d q_{k} \\
& =\sum_{k=1}^{n}( \pm) \int_{q_{k 0}}^{q_{k}}\left[-2 \psi_{k}\left(q_{k}\right)+2 \sum_{i=1}^{n} \phi_{k i}\left(q_{k}\right) \alpha_{i}\right]^{1 / 2} d q_{k},
\end{aligned}
$$

where the sign is \pm respectively as $d q_{k} \gtrless 0$.

\section{Conditionally Periodic Staeckel Systems}

We call a Staeckel system conditionally periodic if each coordinate is either rotational or librational. A coordinate $q_{k}$ is rotational if: $(i)$ it is an angle, (ii) with $p_{k}^{2} \equiv F_{k}\left(q_{k}\right)$ there exist positive real numbers $c_{1 k}$ and $c_{2 k}$ such that $c_{2 k} \geqq F_{k}\left(q_{k}\right) \geqq c_{1 k}>0$ for all real values of $q_{k}$, and (iii) if

$$
F_{k}\left(q_{k}+2 \pi\right)=F_{k}\left(q_{k}\right) .
$$

Note that $c_{1 k}>0$ rules out asymptotic motions and that the periodicity implied by (8) may reduce to simple constancy. The latter holds, e.g., when $q_{k}$ is the right ascension $\phi$ of an artificial satellite, since there does not exist any potential, depending on the right ascension $\phi$, which both leads to separability and remains finite on the polar axis. ${ }^{3}$ For such a rotational coordinate $q_{k}$ either $p_{k}>c_{1 k}^{1 / 2}$ for all $q_{k}$ or $p_{k}<-c_{1 k}^{1 / 2}$ for all $q_{k}$. In either case

$$
v_{k}\left(q_{k}\right) \equiv \int_{q_{k 0}}^{q_{k}} d q_{k} / p_{k}
$$

is a single-valued function of $q_{k}$, with derivative $d v_{k} / d q_{k}$ existing and differing from zero for all values of $q_{k}$. Thus $q_{k}$ is a single-valued differentiable ${ }^{4}$ function of $v_{k}$.

A coordinate $q_{i}$ is librational if there exist real numbers $a_{i}, b_{i}, C_{1 i}$, and $C_{2 i}$ and a real function $G_{i}\left(q_{i}\right)$ such that

with

$$
p_{i}^{2}=\left(q_{i}-a_{\imath}\right)\left(b_{i}-q_{i}\right) G_{i}\left(q_{i}\right),
$$

$$
C_{2 i} \geqq G_{i}\left(q_{i}\right) \geqq C_{1 i}>0 \quad\left(a_{i} \leqq q_{i} \leqq b_{i}\right)
$$

and

$$
a_{i} \leqq q_{i}(0) \leqq b_{i},
$$

$q_{i}(0)$ being the initial value of $q_{i}$. If we again define $v_{i}$ by (9), then

$v_{i}\left(q_{i}\right)= \pm \int_{q_{i 0}}^{q_{i}}\left[\left(q_{i}-a_{i}\right)\left(b_{i}-q_{i}\right) G_{i}\left(q_{i}\right)\right]^{-1 / 2} d q_{i}$,

where the sign is \pm accordingly as $d q_{i} \gtrless 0$, respectively. Then

$$
v_{i}=\int_{E_{i 0}}^{E_{i}} G_{i}^{-1 / 2} d E_{i}
$$

where the uniformizing variable $E_{i}$ is defined by the equation

$$
2 q_{i}=a_{i}+b_{i}+\left(a_{i}-b_{i}\right) \cos E_{i}
$$

and the requirement that $E_{i}$ shall always increase as $q_{i}$ varies. By (12) and (10.2) $v_{i}$ is then a singlevalued function of $E_{i}$, with derivative existing and

${ }^{3}$ See the tables in [9], pp. 656, 658, 660 .

4 Hereafter abbreviated to "s.v.d." 
nonvanishing for all $E_{i}$, so that $E_{i}$ must be a s.v.d. function of $v_{i}$. By (13), however, $q_{i}$ is a s.v.d. function of $E_{i}$, so that, finally, $q_{i}$ is a s.v.d. function of $v_{i}$. Thus in a conditionally periodic Staeckel system any coordinate $q_{k}$ is a s.v.d. function of the corresponding $v_{k}$.

\section{The $v_{k}$ 's as Functions of the Angle-Varia- bles $w_{k}$}

If we now let an increase of $2 \pi$ be one cycle of a rotational coordinate and a single round trip from $a_{k}$ to $b_{k}$ be one cycle of a librational coordinate, we may define the action and angle variables $J_{k}$ and $w_{k}$ by

$$
J_{k} \equiv \oint p_{k} d q_{k}
$$

and

$$
w_{k} \equiv \partial W / \partial J_{k},
$$

where $W$ is now to be considered a function of the $q$ 's and the J's, rather than of the $q$ 's and the $\alpha$ 's. It is well known $[10,11]$ that $J_{k}$ and $w_{k}$ are canonically conjugate, so that

$$
\dot{w}_{k}=\partial \alpha_{1} / \partial J_{k} \equiv \nu_{k} .
$$

If we also define the Jacobi variables $B_{i}$ by

$$
B_{i} \equiv \partial W / \partial \alpha_{i},
$$

we obtain

$$
B_{i}=\sum_{k=1}^{n} \frac{\partial W}{\partial J_{k}} \frac{\partial J_{k}}{\partial \alpha_{i}}=\sum_{k=1}^{n} w_{k} \omega_{k i}
$$

where

$$
\omega_{k i} \equiv \partial J_{k} / \partial \alpha_{i}
$$

Increments $d w_{1}, \ldots, d w_{n}$ then lead to

$$
d B_{i}=\sum_{k=1}^{n}\left(d w_{k}\right) \omega_{k i} \quad(i=1, \ldots, n) .
$$

But, by (7) and (17),

$$
d B_{i}=\sum_{k=1}^{n} \frac{\partial p_{k}}{\partial \alpha_{i}} d q_{k}=\frac{1}{2} \sum_{k=1}^{n} \frac{\partial p_{k}^{2}}{\partial \alpha_{i}} d q_{k} / p_{k}
$$

or

$$
d B_{i}=\sum_{k=1}^{n} \phi_{k i}\left(q_{k}\right) d v_{k}
$$

by (21), (6), and (9). Also, by (19), (14), (6), and (9)

$$
\omega_{k i}=\oint \phi_{k i}\left(q_{k}\right) d v_{k}
$$

If we now introduce the matrix $\Phi$, the matrix $\Omega \equiv\left(\omega_{k i}\right)$, and the row matrices $d v \equiv\left(d v_{1}, \ldots, d v_{n}\right)$ and $d w \equiv\left(d w_{1}, \ldots, d w_{n}\right)$, we find from $(20)$ and (22)

$$
d v \Phi=d w \Omega .
$$

For a nonsingular Staeckel system ${ }^{5} \Phi, \Phi^{-1}$, and $\Omega$ all exist at every point of $Q$, so that

$$
d v=d w \Omega \Phi^{-1}
$$

or

$$
\partial v_{i} / \partial w_{k}=\left(\Omega \Phi^{-1}\right)_{k i} .
$$

Thus each derivative $\partial v_{i} / \partial w_{k}$ exists and is singlevalued everywhere in $Q$. Now the $J$ 's are all real, by (14). Thus, by (7) and (15), if the $p$ 's are all real, then $W$ and the $w$ 's are all real; if some of the $p$ 's are nonreal, then $W$ is nonreal and so are some of the $w$ 's. If all the $w$ 's are real, it then follows that all the $p$ 's are real, else we should have a contradiction. Thus the domain $Q$, corresponding to the totaiity of all real values of the $q$ 's for which the $p$ 's are all real, also corresponds exactly to the set of all possible real values for all the $w$ 's. It therefore follows that each derivative $\partial v_{i} / \partial w_{k}$ exists and is single-valued at any point in $w$-space. Thus each $v_{k}$ must be a s.v.d. function of $w_{1}, \ldots ., w_{n}$. In section 3 , however, we showed that each $q_{k}$ is a s.v.d. function of the corresponding $v_{k}$. Thus for a conditionally periodic nonsingular Staeckel system each $q_{k}$ is a s.v.d. function $f_{k}\left(w_{1}, \ldots, w_{n}\right)$.

\section{Periodic Properties of $q_{k}=f_{k}\left(w_{1}, \ldots, w_{n}\right)$}

By (7) and (15)

$$
d w_{k}=\sum_{i=1}^{n} \frac{\partial p_{i}}{\partial J_{k}} d q_{i}
$$

If now each coordinate $q_{i}$ goes through an integral number $m_{i}$ of cycles, then by a familiar argument

$\Delta w_{k}=\sum_{i=1}^{n} m_{i} \oint\left(\partial p_{i} / \partial J_{k}\right) d q_{i}=\frac{\partial}{\partial J_{k}} \sum_{i=1}^{n} m_{i} \oint p_{i} d q_{i}=m_{k}$.

Thus if each $q_{k}$ goes through exactly $m_{k}$ cycles, each $w_{k}$ increases by the integer $m_{k}$. (Note, however, that such simultaneous increases are not always physically possible: this section is thus concerned only with the mathematical properties of the functions $f_{k}\left(w_{1}, \ldots, w_{n}\right)$.)

But we are really interested in the inverse problem where each $w_{k}$ has increased by an integer $m_{k}$ and we ask what has happened to the $q$ 's. Now the $q$ 's are uniquely determined by the $w$ 's, because of the single-valued property. In the situation of the preceding paragraph where each librational coordinate returns to its initial value and each rotational coordinate $q_{i}$ increases by $2 \pi m_{i}$, each angle variable $w_{k}$ increases by $m_{k}$. Since the $w$ 's determine the $q$ 's uniquely, this has the result that whenever $\Delta w_{k}=$ $m_{k}, k=1$, . . ., $n$, each librational coordinate returns to its initial value and each rotational coordinate $q_{i}$ increases by $2 \pi m_{i}$.

\footnotetext{
5 See appendix for examples.
} 
Thus, in the inverse problem, whenever we are given $\Delta w_{k}=m_{k}, k=1, \ldots ., n$, we find that each rotational coordinate $q_{i}$ must go through exactly $m_{i}$ cycles and that each librational coordinate $q_{j}$ must go through some integral number of cycles, $\tau_{j}$, say. By (28), however, we then find $\tau_{j}=m_{j}$. Thus whenever the angle variables $w_{k}$ are all increased by integer amounts $\Delta w_{k}=m_{k}$, each of the functions $q_{k}=f_{k}\left(w_{1}, \ldots, w_{n}\right)$ must go through exactly $m_{k}$ cycles.

\section{Mean Motions}

If in a time interval $T$ the number of complete cycles passed through by any coordinate $q_{k}$ is $N_{k}$, the corresponding mean frequency $n_{k}$ is, by definition

$$
n_{k}=\lim _{T \rightarrow \infty}\left(N_{k} / T\right),
$$

if the limit exists. We shall now prove that $n_{k}=\nu_{k} \equiv \partial \alpha_{1} / \partial J_{k}, \quad k=1, \ldots, n$, for any conditionally periodic nonsingular Staeckel system.

To do so, note that if $\nu_{1}, \ldots, \nu_{n}$ are all commensurable, there exist a positive $\nu_{0}$ and positive integers $m_{1}, \ldots, m_{n}$ such that

$$
\nu_{k}=m_{k} \nu_{0}, \quad(k=1, \ldots, n),
$$

where we may choose $\nu_{0}$ to be the greatest common divisor of the $\nu_{k}$ 's. Then, from (16) and (30), during the actual motion,

$$
w_{k}=w_{k}(0)+m_{k} \nu_{0} t \quad(k=1, \ldots, n)
$$

and in the time interval $T \equiv 1 / \nu_{0}$ we have

$$
\Delta w_{k}=m_{k} \quad(k=1, \ldots, n) .
$$

By section 5 each $q_{k}$ goes through exactly $m_{k}$ cycles in this time, so that in this case the motion is truly periodic, with period $1 / \nu_{0}$. The mean frequency of $q_{k}$ is thus

$$
n_{k} \equiv m_{k} / T=m_{k} \nu_{0}=\nu_{k} .
$$

If the frequencies $\nu_{1}, \ldots, \nu_{n}$ are not all commensurable, we may let

$$
\xi_{k} \equiv \nu_{k} / \nu_{1} \quad(k=1, \ldots, n)
$$

and then at least one of the $\xi$ 's will be irrational. Then by (16) and (34), during the actual motion

$$
w_{k}=w_{k}(0)+\xi_{k} \nu_{1} t .
$$

We now use a theorem of Dirichlet [12], which states that if the set of real numbers $\xi_{1}, \ldots, \xi_{n}$ has at least one irrational member, then the system of inequalities

$$
\left|\xi_{k}-m_{k} / P\right|<P^{-1-\frac{1}{n}} \quad(k=1, \ldots, n)
$$

has an infinite number of integer solutions for $P$ and the $m$ 's. Note that the solutions for $P$ have no upper bound.

To apply this theorem, consider only those values of the time interval $T$ such that $\nu_{1} T=P$, where $P$ is an integer that satisfies (36). In this time each $w_{k}$ increases from its initial value $w_{k}(0)$ to a final value given by

$$
w_{k}(T)=w_{k}(0)+P \xi_{k},
$$

by (35). But by (36)

so that

$$
P \xi_{k}=m_{k}+\eta_{k}, \quad\left|\eta_{k}\right|<P^{-\frac{1}{n}}
$$

$$
w_{k}(T)=w_{k}(0)+m_{k}+\eta_{k} .
$$

As $\nu_{1} T=P$ takes on those larger and larger integer values corresponding to solutions of (36), each $\eta_{k}$ approaches zero, by (38). Then, by (39), there exist infinitely many values of $T$, with no upper bound, at which the orbit in $w$-space passes arbitrarily closely to points where $\Delta w_{k}=m_{k}, k=1, \ldots, n$, the $m_{k}$ 's being solutions of (36).

If the initial $q$ 's are given by

$$
q_{k}(0)=f_{k}\left[w_{1}(0), \ldots, w_{n}(0)\right], \quad(k=1, \ldots, n),
$$

then the values of the $q$ 's at any of these times $T$ are given by

$$
\begin{array}{r}
q_{k}(T)=f_{k}\left[w_{1}(0)+m_{1}+\eta_{1}, \ldots,\right. \\
\left.w_{n}(0)+m_{n}+\eta_{n}\right] \\
(k=1, \ldots, n) .
\end{array}
$$

As we let $T=P / \nu_{1}$ assume those larger and larger values already referred to, the $q$ 's then approach arbitrarily closely to the values

$$
\begin{array}{r}
q_{k}^{*}(T)=f_{k}\left[w_{1}(0)+m_{1}, \ldots, w_{n}(0)+m_{n}\right] \\
(k=1, \ldots, n) .
\end{array}
$$

This conclusion follows from (38) and the singlevaluedness and differentiability of the functions $f_{k}$.

Comparison of (40) and (42) then shows that the values $q_{k}^{*}(T)$ correspond to $\Delta w_{k}=m_{k}, k=1, \ldots, n$, and are thus, by section 5 , the values that would be reached after each $q_{k}$ had gone through exactly $m_{k}$ cycles. Now, by the definition (29), it follows that the mean frequency

$$
n_{k}=\lim _{T \rightarrow \infty}\left(m_{k} / T\right),
$$

if the limit exists. But $m_{k} / T=\nu_{1} m_{k} / P$ and, as $T \rightarrow \infty, \lim \left(m_{k} / P\right)=\xi_{k}$, by (36). Thus,

$$
n_{k}=\nu_{1} \xi_{k}=\nu_{k}
$$

by (43) and (34).

Thus, for each coordinate $q_{k}$ of a conditionally periodic nonsingular Staeckel system, the mean frequency $n_{k}$ is equal to the corresponding fundamental frequency $\nu_{k} \equiv \partial \alpha_{1} / \partial J_{k}$. 


\section{Appendix}

For theories of satellite orbits, appropriate coordinates are spherical or oblate spheroidal. The corresponding Staeckel matrices and their inverses are, if $x=r \sin \theta \cos \phi, y=r \sin \theta \sin \phi, z=r \cos \theta$ :
$\Phi=\left(\begin{array}{ccc}1 & -r^{-2} & 0 \\ 0 & 1 & -\csc ^{2} \theta \\ 0 & 0 & 1\end{array}\right) \Phi^{-1}=\left(\begin{array}{ccc}1 & r^{-2} & r^{-2} \csc ^{2} \theta \\ 0 & 1 & \csc ^{2} \theta \\ 0 & 0 & 1\end{array}\right)$

or, if $x=c\left[\left(\xi^{2}+1\right)\left(1-\eta^{2}\right)\right]^{1 / 2} \cos \phi, \quad y=c\left[\left(\xi^{2}+1\right)\left(1-\eta^{2}\right)\right]^{1 / 2} \sin \phi, \quad z=c \xi \eta$ :

$$
\begin{gathered}
\text { Oblate Spheroidal } \\
\Phi=\left(\begin{array}{ccc}
c^{2} \xi^{2}\left(\xi^{2}+1\right)^{-1} & -\left(\xi^{2}+1\right)^{-1} & \left(\xi^{2}+1\right)^{-2} \\
c^{2} \eta^{2}\left(1-\eta^{2}\right)^{-1} & \left(1-\eta^{2}\right)^{-1} & -\left(1-\eta^{2}\right)^{-2} \\
0 & 0 & 1
\end{array}\right) \\
\Phi^{-1}=\left(\begin{array}{ccc}
c^{-2}\left(\xi^{2}+1\right)\left(\xi^{2}+\eta^{2}\right)^{-1} & c^{-2}\left(1-\eta^{2}\right)\left(\xi^{2}+\eta^{2}\right)^{-1} & c^{-2}\left(\xi^{2}+1\right)^{-1}\left(1-\eta^{2}\right)^{-1} \\
-\eta^{2}\left(\xi^{2}+1\right)\left(\xi^{2}+\eta^{2}\right)^{-1} & \xi^{2}\left(1-\eta^{2}\right)\left(\xi^{2}+\eta^{2}\right)^{-1} & \left(\xi^{2}+\eta^{2}\right)^{-1}\left[\xi^{2}\left(1-\eta^{2}\right)^{-1}+\eta^{2}\left(1+\xi^{2}\right)^{-1}\right] \\
0 & 0 & 1
\end{array}\right)
\end{gathered}
$$

For spherical coordinates $\Phi$ is most easily written down from the expressions for $p_{r}^{2}, p_{\theta}^{2}$, and $p_{\phi}^{2}$ in the Kepler problem [1], with replacement of $\alpha_{2}^{2}$ and $\alpha_{3}^{2}$ by $2 \alpha_{2}$ and $2 \alpha_{3}$. For oblate spheroidal coordinates $\Phi$ may be found by comparing eqs (53) and (59.1) of [5] with eq (6) of the present paper.

Note that $\Phi$ or $\Phi^{-1}$ could fail to exist only when $\sin \theta=0$ or when $\eta^{2}=1$. This could happen only when the satellite goes over a pole and thus only in a polar orbit. Such a singularity in a polar orbit, however, is to be expected, since nonsingularity of a Staeckel system leads to the $q$ 's being differentiable functions of the $w$ 's and thus of the time. In a polar orbit, on the other hand, the right ascension $q_{3} \equiv \phi$ is a discontinuous function of time, being constant except at polar crossings, where it changes by $\pi$.
(Paper 65B2-52)

\section{References}

[1] W. M. Smart, Celestial Mechanics, pp. 143-148 (Longmans, Green, \& Co., New York, N.Y., 1953).

[2] C. L. Charlier, Die Mechanik des Himmels I (Veit and Co., Leipzig, Germany, 1902).

[3] T. E. Sterne, Astron. J. 62, 96 (1957); 63, 28-40 (1958).

[4] B. Garfinkel, Astron. J. 63, 88-96 (1958); 64, 353-367 (1959).

[5] J. P. Vinti, Phys. Rev. Letters 3, 8 (1959); J. Research NBS 63B, 105-116 (1959).

[6] P. Staeckel, Habilitationsschrift (Halle, 1891).

[7] L. P. Eisenhart, Annals of Math. 35, 284-305 (1934).

[8] L. P. Eisenhart, Phys. Rev. 74, 87-89 (1948).

[9] P. M. Morse and H. Feshbach, Methods of Theoretical Physics I, 509-518, 655-664'(McGraw Hill Book Co., Inc., New York, N.Y., 1953).

[10] P. Frank and R. v. Mises, Die Differential-und Integralgleichungen der Physik II, 94-101 (Vieweg \& Sohn, Braunschweig, Germany, 1935).

[11] M. Born, The Mechanics of the Atom, ch. 1, 2 (G. Bell and Sons, Ltd., London, England, 1927).

[12] G. H. Hardy and E. M. Wright, An Introduction to the Theory of Numbers, p. 169, theorem 200 (Clarendon Press, Oxford, England, 1938). 